\title{
Applications of Single Sleeper Method in Submarine Pipeline Buckling
}

\author{
Liu Wenbin \\ CCCC Tianjin Port Engineering Institute Co., Ltd. \\ Tianjin, China \\ Liu Aimin \\ CCCC Tianjin Port Engineering Institute Co., Ltd. \\ Tianjin, China
}

\begin{abstract}
Global buckling of submarine pipeline in high temperature and high pressure has become the key problem in pipeline stability design, which restricts the development of offshore oil and gas resource. Due to the pipeline is full of oil and gas, once to destroy or leak, the state economy and environment would suffer great destructions, therefore, the methods of pipeline global buckling protection are popular in design and constructions. Base on the pipeline project of Angola oil field in West Africa Sea, this paper designed a single sleeper method to control the pipeline global buckling and monitored the implementation effects. According to the monitor results, the factors are researched on influencing the pipeline stress.
\end{abstract}

Keywords-submarine pipeline; high temperature; high pressure; global buckling; sleeper method

\section{INTRODUCTION}

Owing to human factors or the condition of uneven seabed, a local bend is produced in the process of pipeline manufacturing and laying which is called as general initial imperfection and make the submarine pipeline can't on the seabed straightly. Taylor and Gan[1]considered soil resistance in the pipeline deformation process and deduced the analytical solution for the first - and second - order buckling models of submarine pipeline, which had geometric initial imperfection in the vertical or lateral direction. Taylor[2] provided three different models of pipeline imperfection based on the theory and experimental research on vertical buckling and obtained the calculation equation for critical buckling load, but didn't take the influence of residual stress caused by geometric initial imperfection on the global buckling. Junes [3] established nonlinear finite element models for buried pipelines and simulated the vertical buckling of submarine pipeline with geometric imperfection considering nonlinear constraints of soil foundation. David[4] revealed the global buckling mechanism based on nonlinear finite element analysis and emphasized that the axial force on internal pipeline, geometric initial imperfection of pipeline, and soil resistance are the main factors controlling the buckle shape. Yu [5] utilized 2D and 3D numerical analysis methods to study the pipe-soil interaction in lateral buckling and the influence of pipeline diameter and weight on buckling models. In past numerical analysis, the geometric initial imperfection of

\author{
Ye Guoliang * \\ CCCC Tianjin Port Engineering Institute Co., Ltd. \\ Tianjin, China \\ e-mail: yeggll@sohu.com \\ * Corresponding Author \\ Cao Yonghua \\ CCCC Tianjin Port Engineering Institute Co., Ltd. \\ Tianjin, China
}

pipeline is introduced by drawing the size of amplitude and wavelength to confirm the features of geometric imperfection. Many research scholars, such as Yu [5]. Base on the pipeline project of Angola oil field in West Africa Sea, the paper monitored the implementation effects of signal sleeper method, provided a large of basis dates for controlling pipeline global buckling.

\section{CASE STUDIES}

The operating depth of Angola oil field in West Africa Sea is $1150-1500 \mathrm{~m}$, the general arrangement of the oil field is in Figure1.

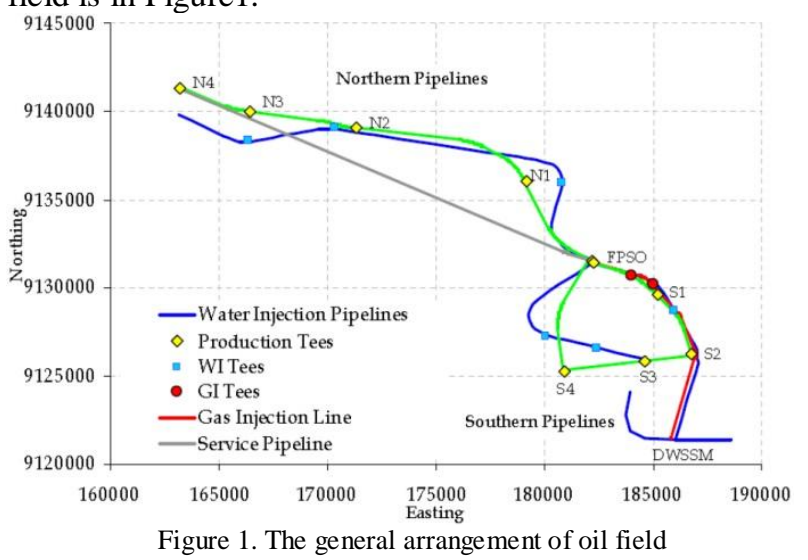

There are two pipeline systems in the project, which is south pipeline system, and north pipeline system. The paper mainly analyzes the $\mathrm{S} 1$ pipeline of south pipeline system and the pipeline's detail characters are shown in Table 1.

Table I Design Parameters

\begin{tabular}{|l|l|l|}
\hline Parameters & Values & Unit \\
\hline External diameter & 323.9 & $\mathrm{~mm}$ \\
\hline Wall thickness & 19.1 & $\mathrm{~mm}$ \\
\hline Elastic modulus & 206 & $\mathrm{GPa}$ \\
\hline Steel density & 7850 & $\mathrm{~kg} / \mathrm{m}^{3}$ \\
\hline $\begin{array}{l}\text { Coefficient of thermal } \\
\text { expansion }\end{array}$ & $1.1 \times 10^{-5}$ & $1{ }^{\circ} \mathrm{C}$ \\
\hline Poisson ratio & 0.3 & --- \\
\hline Lateral friction & 0.4 & --- \\
\hline yield strength & 520 & $\mathrm{MPa}$ \\
\hline
\end{tabular}


In order to control the random global buckling of submarine pipeline, two single sleepers are respectively set on the ends of the pipeline. The sleeper's height is $1 \mathrm{~m}$ and spacing is $500 \mathrm{~m}$, as shown in Figure 2.
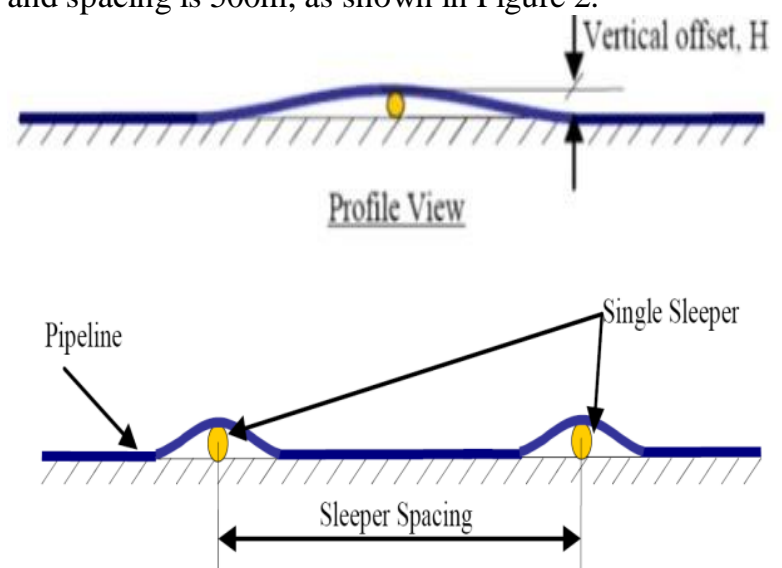

Figure 2. single sleepers' laying

The sleepers make the pipeline form a vertical initial deformation and reduce the critical load of pipeline global buckling. Once the temperature increase to a certain value, the axial pressure of pipeline firstly achieve to the buckling load, which make the pipeline global buckling to release the axial pipeline pressure and guarantee the pipeline's on side stability.

\section{MONITOR RESULTS}

Monitor and analyze the operation condition of pipeline. Figure 3 is drawing the pipeline global buckling shape with laying a single sleeper on the bottom. In Fig .3.the structure in yellow is stand for the sleeper, the straight line in gray is stand for the pipeline installing location and the structure in red is stand for the pipeline after global buckling.

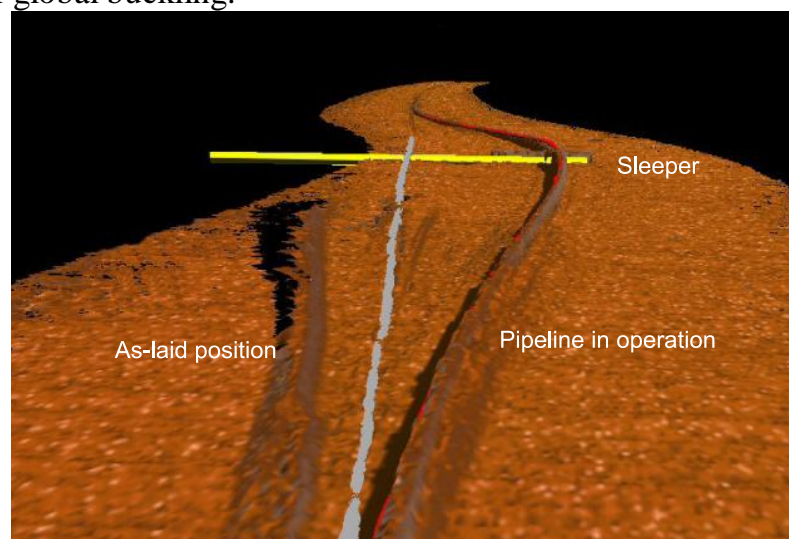

Figure 3. Global buckling of pipeline with laying a single sleeper on the bottom

Fig .4 shows the survey data of pipeline's out of straightness in installation, production and operation stages.

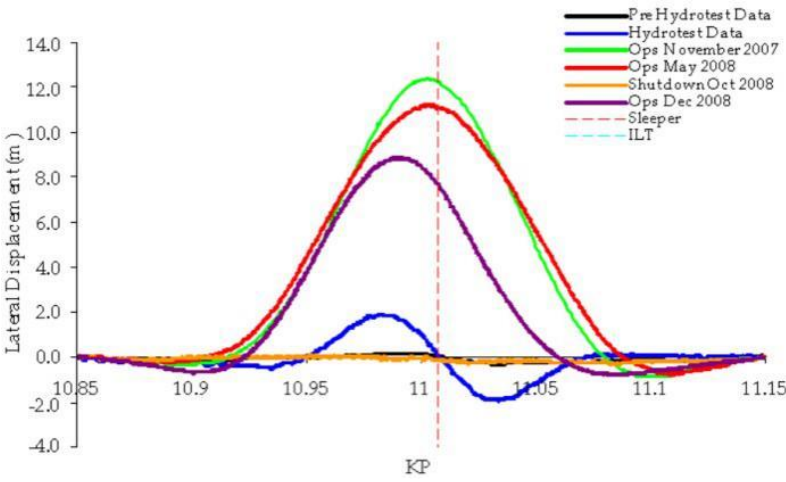

Figure 4 .The survey data of pipeline's out of straightness

Fig .4 shows that the pipeline which laying a sleeper on the bottom is a straight line on the stage of pipeline. On the stage of pre-pressure, there is a tiny deformation on the pipeline; on the stage of pressure, there is a large lateral buckling deformation on pipeline in predicting value range. On the first operation stage, the deformation's models and values are all in control; when the pipeline stops to operate, the pipeline recover to flat and straight state; after restart, the pipeline is in buckling predict condition and moves globally.

\section{CONCLUSIONS}

Laying a sleeper on the predict location of pipeline can control the deformation of pipeline global buckling and release the axial pressure to guarantee the on side stability. The value of buckling stress is related to the value of transport medium's density, the greater density of the medium, the greater pipe stress is.

\section{REFERENCES}

[1] Taylor, N, and Gan, AB (1986). "Submarine pipeline buckling-imperfection studies," Thin-Walled Structures, Vol 4, No 4, pp 295-323.

[2] Taylor, N, and Tran, V (1996). "Experimental and theoretical studies in subsea pipeline buckling," Marine Structures, Vol 9, No 2, pp 211-257.

[3] Junes A V, Jose F. R, Cora M, Buried Pipe Modeling with Initial Imperfections [J]. Journal of Pressure Vessel Technology, 2004, 126(2):250-257.

[4] David A S Bruton, AtkinsBoreas, David J White, Pipe-Soil Interaction During Lateral Buckling and Pipeline Walking-The SAFEBUCK JIP, Offshore Technology Conference, 2008, 1-20.

[5] Yu S.K., Konuk I., Continuum FE modeling of lateral buckling: Study of soil effects[C], Proceedings of the International Conference on Offshore Mechanics and Arctic Engineering OMAE, 2007, 347-354. 